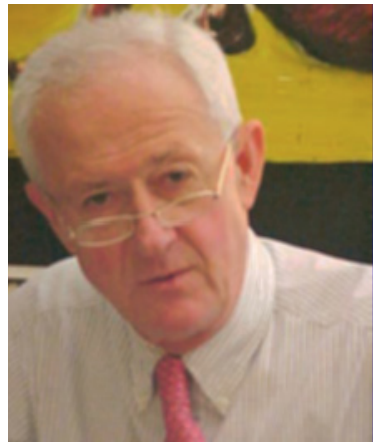

-Daniel Serin

Rédacteur en chef

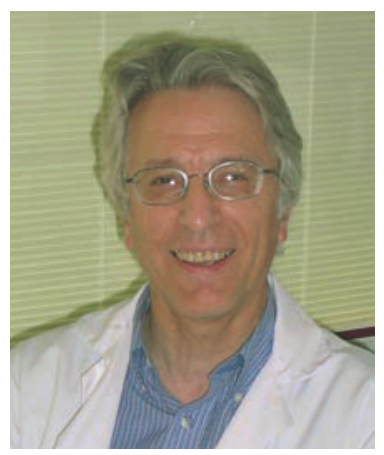

Pierre Marti Rédacteur en chef adjoint

\title{
Alerte en cancérologie !
}

En cancérologie - comme ailleurs mais plus encore - le patient doit être au centre de la prise en charge. Le médecin généraliste est le " pivot » du système de santé et doit jouer ici le rôle de "coordonnateur ». Les proches sont, comme leur nom l'indique, près du patient mais aussi autour puisqu'ils l'entourent. Le patient est un centre qui se déplace puisqu'il connaît de nombreux parcours parmi lesquels le «parcours nutrition ». Comme on le voit, la géographie du cancer est pour le moins complexe.

Améliorer la prise en charge des patients ne nécessite pas forcément toujours plus de moyens : D. Delfieu dresse le tableau du médecin généraliste face des impératifs administratifs parfois ubuesques. 31 patients vus dans une journée pour 223 coups de tampon ! Mercre alors !

J.P. Escande attire notre attention sur les proches qui, comme les médecins généralistes, sont de plus en plus sollicités - quand ce n'est pas culpabilisés-, sans que leur propre souffrance ou leur épuisement soient toujours pris en compte. Cette " mobilisation » de toutes les énergies (proches, médecins généralistes, etc.) est d'autant plus indispensable qu'il s'agit de « combattre un fléau ». Fléau, dont B. Hoerni se demande s'il n'est pas avant tout un témoin de notre humaine condition?

Le patient au domicile est entouré par ses proches, a comme interlocuteur premier son médecin généraliste (ou mieux son médecin de famille) et baigne dans un ensemble de représentations qui fait du cancer un fléau.

Ce sont donc trois articles en prise sur la réalité quotidienne que nous livrent les auteurs.

Le cancer un fléau ? En tous les cas une injustice pour Julia Domna, Syrienne et impératrice de Rome. D. Gros illustre une fois encore la permanence des attitudes humaines à travers les siècles : le cancer conséquence d'un coup ? Lié aux malheurs de la vie (aujourd'hui au stress) ? Sont-ce des idées modernes ? Non ! Ce sont déjà celles de Julia Domna au Ile siècle.

Les multiples interfaces générées par la mise en œuvre de traitements autres que spécifiques ont conduit à la naissance de l'AFSOS (Association française pour les soins oncologiques de support) que son président I. Krakowski nous présente ici.

L. Mineur nous fait part de son expérience dans la prise en charge nutritionnelle des patients ; expérience qui va bien au-delà du simple conseil nutritionnel et intègre éducation des patients et formation des professionnels.

Pour conduire toutes ces actions et améliorer encore et toujours la prise en charge des patients, il faut des hommes et des femmes compétents.

Toutefois, il ne convient pas de déshabiller Pierre pour habiller Paul, et l'augmentation du nombre de praticiens dans un domaine de la prise en charge ne doit pas se faire aux dépens d'un autre. À cet égard, I'anatomopathologie est une discipline indispensable à la pratique de l'oncologie et l'évolution de la démographie médicale est inquiétante. F.Penault-Llorca nous alerte sur le petit nombre de praticiens formés, la complexité croissante des demandes et la non-prise en compte de leurs répercussions économiques.

Au-delà de la situation quasi paradigmatique de l'anatomopathologie, c'est l'évolution de l'oncologie médicale toute entière qui fait question. L'appel lancé à l'initiative de D. Serin par les oncologues médicaux au Président de la République tend à sensibiliser les plus hautes autorités de l'État à cette problématique alors que le $2^{\text {e }}$ Plan Cancer voit le jour.

En souhaitant ne pas être des Cassandres !

Bonne lecture! •

Pierre Marti, Daniel Serin

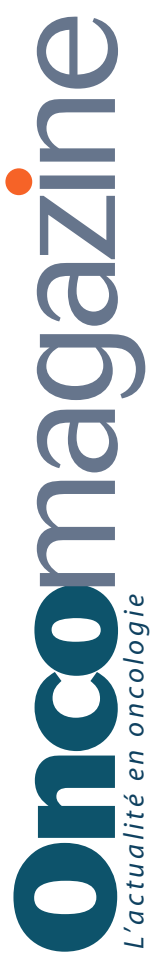

